\title{
Novel 8-Port Network
}

\author{
Eng. W. M. ALSAMKARI ${ }^{1 *}$, Prof. Adnan M. AFFANDI ${ }^{2}$ \\ Bachelor of Electronics and Communications, Department of Electrical and Computer \\ Engineering, King Abdulaziz University, Saudi Arabia ${ }^{1}$
}

Professor, Department of Electrical and Computer Engineering, King Abdulaziz University, Saudi Arabia ${ }^{2}$

*Email: Walsamkari@gmail.com

\begin{abstract}
:
The proposed 8-port network which consists of 4-coupler combined together in unique way to produce 8-port network. This network with the proper terminations will find a lot of applications in microwave and millimeter wave networks.

This proposed 8-port network (with the proper termination) can be used as power combiner divider, six-port reflect meter in order to measure both the amplitude and the phase of the network under test.

The proposed 8-port also can be used as phase comparator in a monopulse radar system for determining both the azimuth and the elevation of the target (K. Chang, et al, 1987). In addition, the circuit can be utilized as a 4-way power combiner/divider with the sub-arms isolated from one another.

Generally, this 8-port network enjoys a compact structure which contains slots in sections the ground plane and between the coupled lines in order to improve the performance of this networks.
\end{abstract}


Also this circuit can be utilized with proper termination as a network synthesize (G. Matthaei, et al, 1980).

In the theoretical analysis of the proposed 8-port network, unique signal flow has been developed for this proposed. The purpose of the 8-port has been fabricated on Roger substrate of dielectric constant $(\varepsilon r=3.38)$ and (thickness of the substrate is $=0.2$ ).

Keyword: Novel, 8-Port Network

\section{Introduction}

A novel 8-port network is used in many microstrip applications such as oscillators, filters (P. Troughton, 1969). This paper introduces a modification to the symmetric square ring resonator described by C. E. Saavedra to develop an eight port network (J. Sewter and C. E. Saavedra, 2002). The network is composed of four quarter-wave couplers, one for each side of the ring. It exhibits resonance at the harmonic frequencies. In this paper, the effect of reducing the separation between the coupled lines is investigated.

Generally, multi ports network finds a lot of applications in microwaved, millimeter and submillimeter devices (C. E. Saavedra, 2001).

Incentive works and researches have been conducted on multi-port especially in 8-port network for their unique applications. Some of these applications are mentioned in references (F. Bray, et al, 2013), (M. Miyakawa, et al, 2004), (E. Praveen, et al, 2016).

In this paper, we consider a circuit constructed with four branch-line 3-dB couplers. First, after deriving admittance relations between the branch line sections for a matching and equal power splitting condition of a basic construction. The frequency dependence of the scattering parameters of this proposed network is calculated. The bandwidth and the isolation of this 8-port are enhanced (i.e. the isolation is more than $-20 \mathrm{~dB}$ is obtained). Finally, we obtain experimental corroboration by measuring the scattering parameters of a band widened circuit. The present circuit can be manufactured in the some plane using a planer transmission line. 


\section{Design Methodology:}

The design process was broken into two major stages. First, a quarter-wave coupler was designed based on the resonator described in. Then, the separation between the coupled lines was reduced to $0.1 \mathrm{~mm}$ and its effect on insertion loss was studied. All circuits were designed for fabrication on a Roger 4003 substrate, with a relative dielectric constant of 3.38 and a thickness of $0.2 \mathrm{~mm}$.

\section{Corrugated Coupler Design and Optimization}

The overall coupler dimensions are equal to those of a traditionally-designed quarter-wave coupler. The separation between the lines is $0.1 \mathrm{~mm}$. The coupler is corrugated along its coupled edges using an alternating square pattern. The corrugations are periodic and symmetric about the midpoint of the lines. The separation distance of $0.1 \mathrm{~mm}$ between coupled edges is maintained at all times along the corrugation.

Sewter designed a square ring resonator from four corrugated quarter-wave couplers and achieved $-30 \mathrm{~dB}$ rejection of harmonics. Two of the couplers were used to couple energy into and out of the ring. The remaining two couplers maintained the symmetry of the device and eliminate the splitresonant behavior of the network. The mitered bends are used to connect the couplers together in order to increase the circumference of the network and to reduce the resonant frequency H. Zhang, et al, 2013).

In this work, the resonator is reduced by the separation between the coupled lines is made to be $0.1 \mathrm{~mm}$ in order to increase the coupling and connected eight $50 \Omega$ ports to convert this resonator into an eight port network. The layout of the network and the prototype circuit implementation on Roger 4003 substrate is shown in fig. 1.

\section{Measurements}

The novel 8-port network is constructed from corrugated quarter-wave couplers. Initially, the dimensions were taken as mentioned before with the separation between the coupled lines as 12 mil. The layout and frequency response of the circuit is shown in figures 1 and 2. 


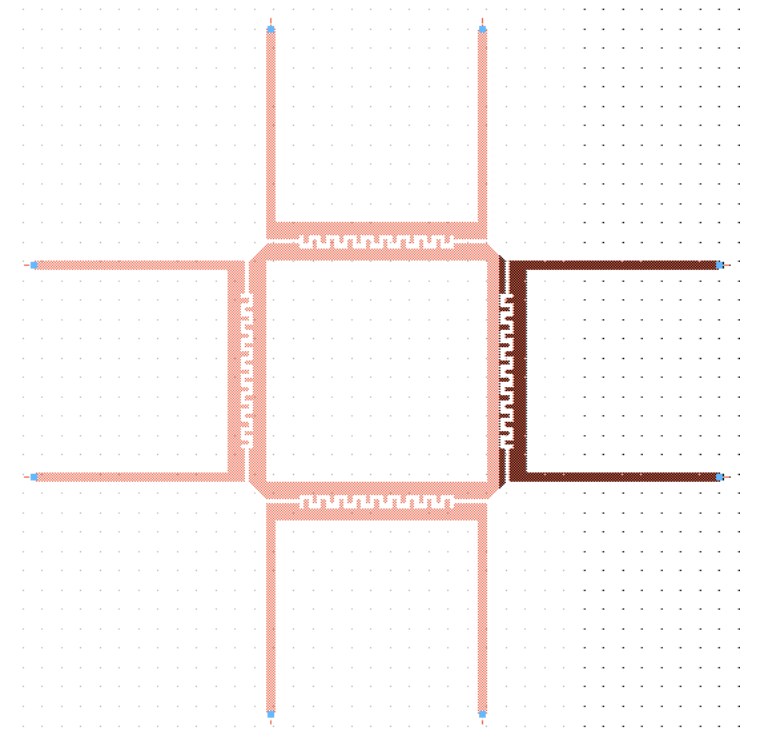

Fig. 1: 8-Port branch line coupler initial design layout diagram

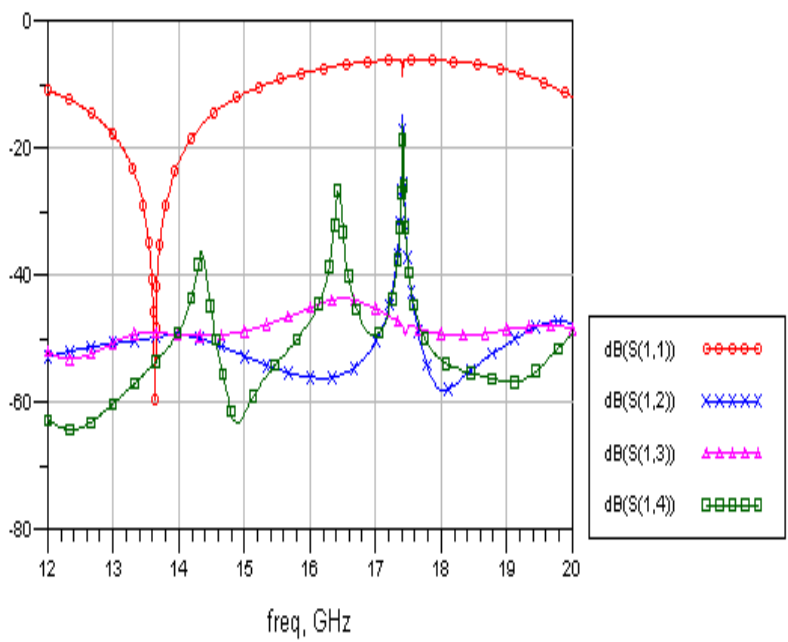

Fig. 2: Frequency response simulated using ADS 2009. 


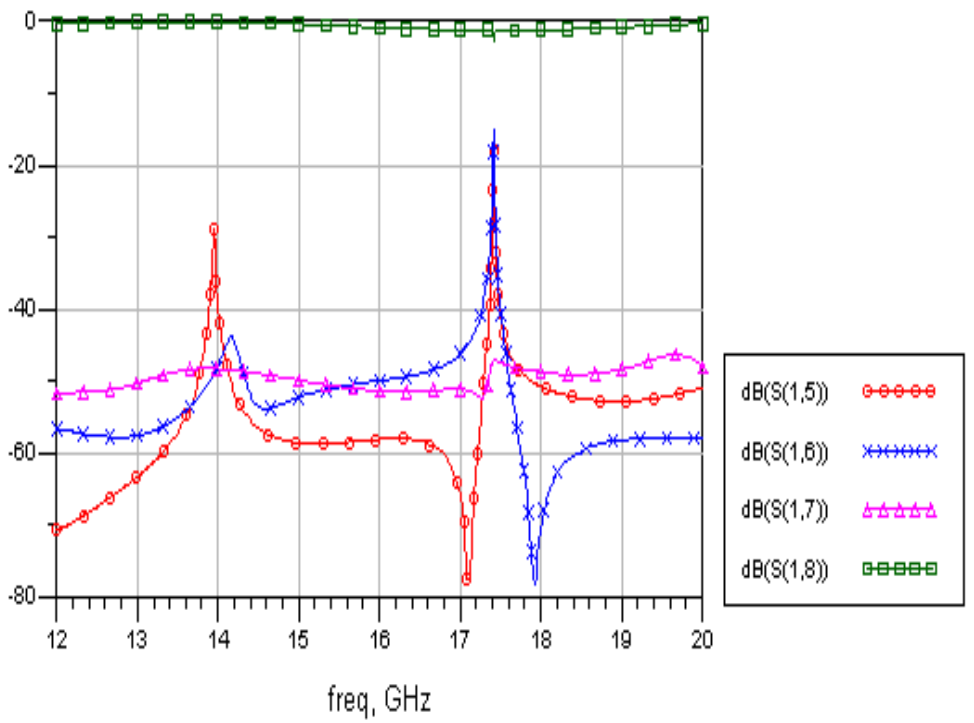

Fig. 3: Shows the frequency response of simulation using ADS.

We observe that the insertion loss is $18 \mathrm{~dB}$ at the resonant frequency which is very high for a coupler. Next we modify the coupler by reducing the separation between the coupled lines to 4 mils $(0.1 \mathrm{~mm})$ (S. Padhi, et al, 2007). The layout and frequency response of the modified circuit is given by Fig. 3 and 4.

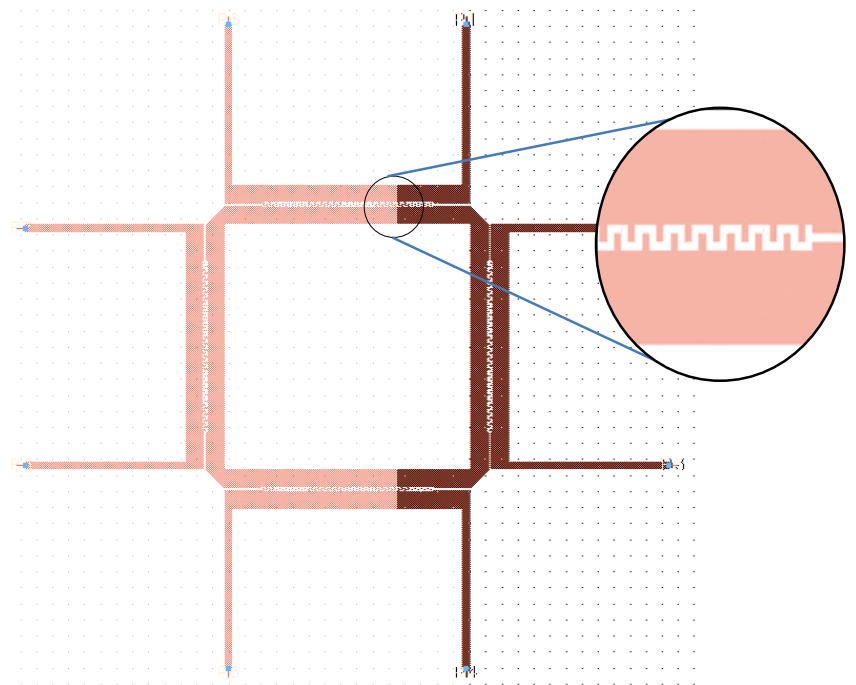

Fig. 4: Modified version of the 8 port branch line coupler layout 
Publication Date: 5-11-2021 ISSN: 2706-6495

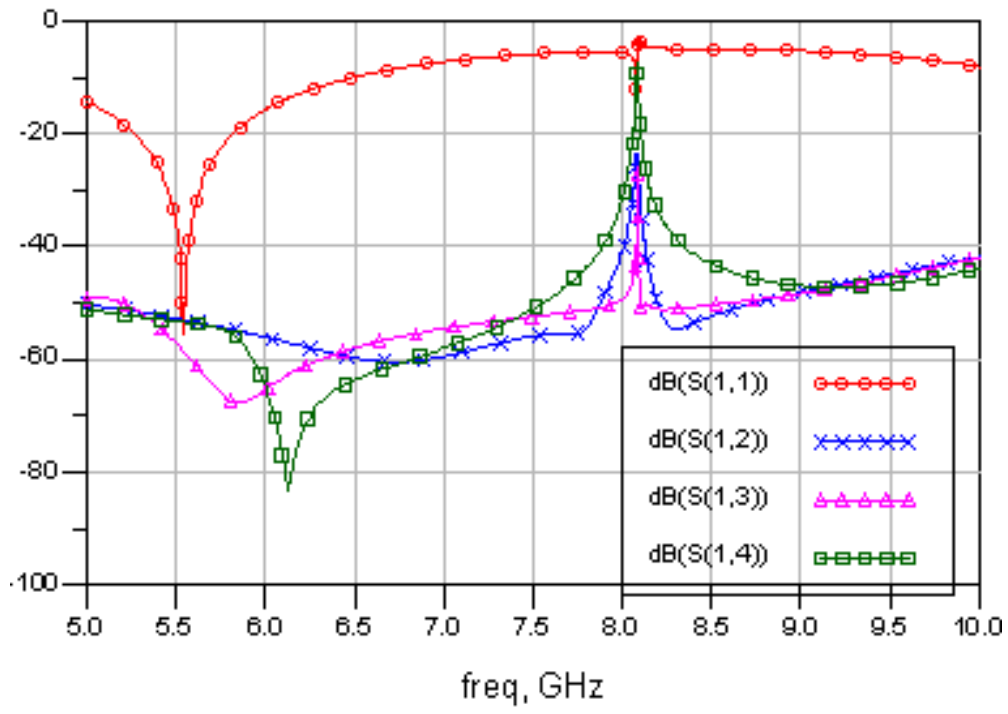

Fig. 5: Frequency Response

Fig.(2,3,4 and 5 show the frequency responses of this proposed 8-port network. This clear from the frequency responses that the manufacture 8-ports has very encourging responses according to the design procedure.

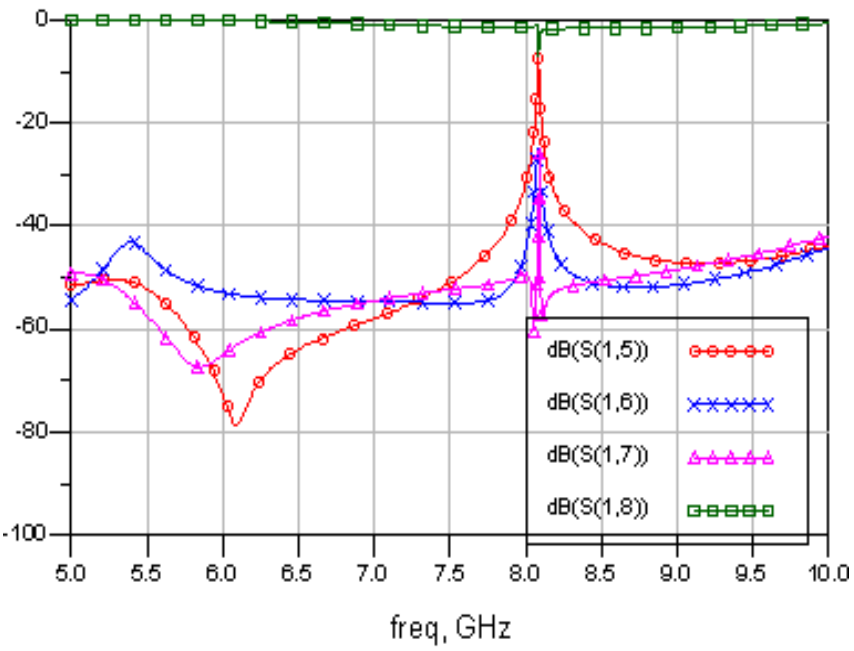

Fig. 6: Frequency Response

The observed insertion loss at the resonant frequency of $8.2 \mathrm{GHz}$ is reduced to $8 \mathrm{~dB}$, which indicates a $10 \mathrm{~dB}$ improvement. 
Academic Journal of Research and Scientific Publishing | Vol 3 | Issue 31

Publication Date: 5-11-2021 ISSN: 2706-6495

\section{Designing:}

Figs. (7) And (8) show the proposed novel 8-port which have been fabricated on Roger substrate of dielectric constant ( $\varepsilon r+=3.2)$ (M. T. Islam, et al, 2019).

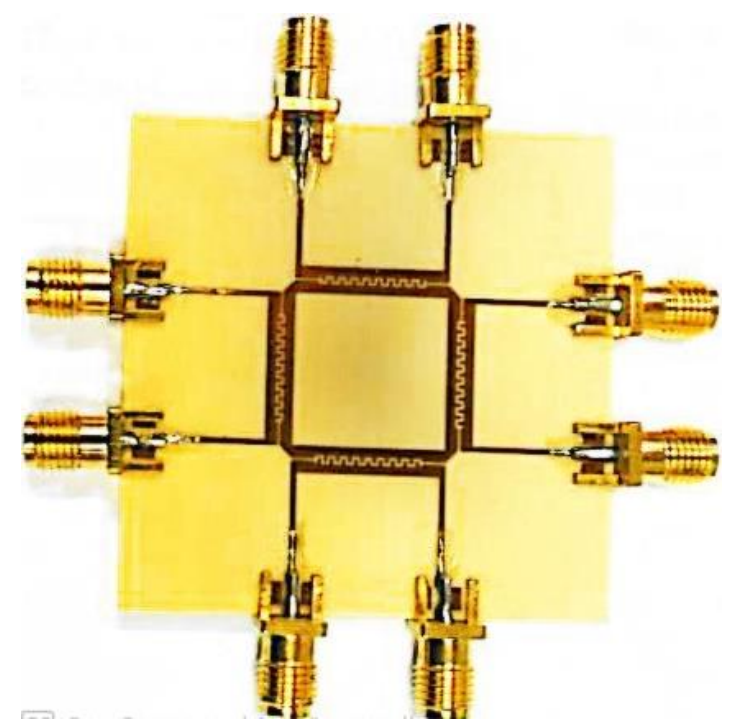

Fig.7

Fig(7) and (8) shows the proposed manufacture 8-port network

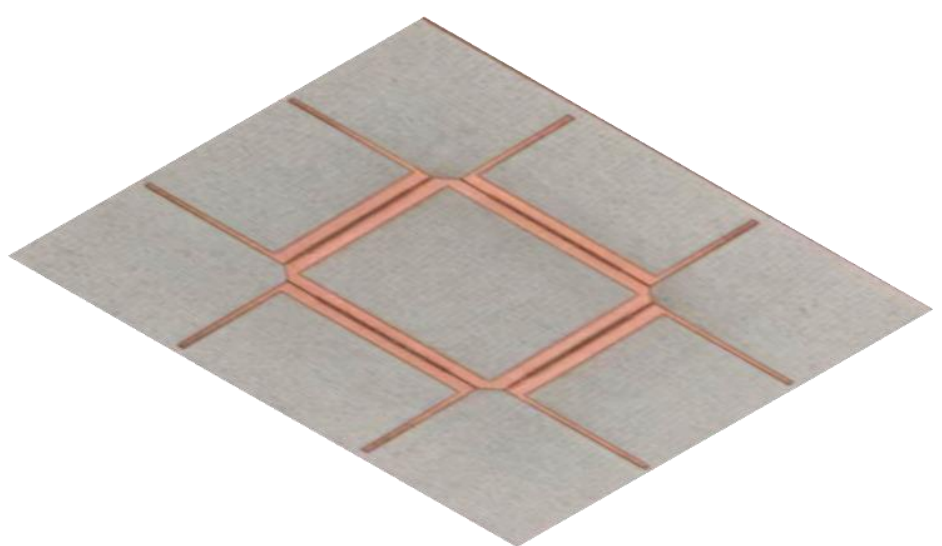

Fig. 8: Prototype circuit printed on Roger substrate 
Academic Journal of Research and Scientific Publishing | Vol 3 | Issue 31

Publication Date: 5-11-2021 ISSN: 2706-6495

\subsection{Signal flow}

The developed signal flow for this proposed 8-port network is analyzing the theoretical analysis of the novel 8-port network, unique signal flow have been developed for this propose in order to analyze theoretically the proposed 8-port theoretically.

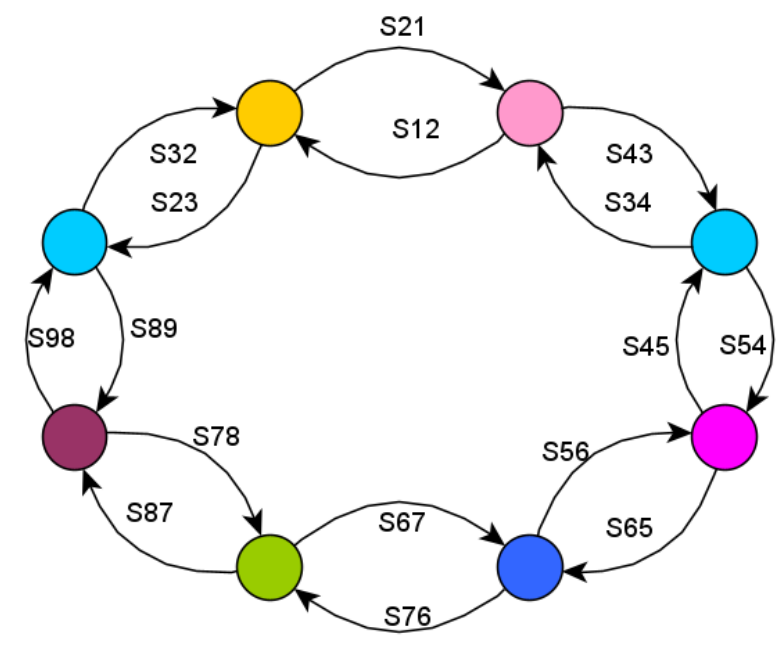

Fig. 9: Signal Flow

Fig (9) shows the developed signal flow, which has the ability to calculate the scattering parameters of the proposed of this network.

\subsection{Scattering Matrix}

$$
\left[\begin{array}{l}
v_{1}^{-} \\
v_{2}^{-} \\
v_{3}^{-} \\
v_{4}^{-} \\
v_{5}^{-} \\
v_{6}^{-} \\
v_{7}^{-} \\
v_{8}^{-}
\end{array}|=| \begin{array}{llllllll|l}
s_{11} & s_{12} & s_{13} & s_{14} & s_{15} & s_{16} & s_{17} & s_{18} & v_{1}^{+} \\
s_{21} & s_{22} & s_{23} & s_{24} & s_{25} & s_{26} & s_{27} & s_{28} & v_{2}^{+} \\
s_{31} & s_{32} & s_{33} & s_{34} & s_{35} & s_{36} & s_{37} & s_{38} & v_{3}^{+} \\
s_{41} & s_{42} & s_{43} & s_{44} & s_{45} & s_{46} & s_{47} & s_{48} & v_{4}^{+} \\
s_{51} & s_{52} & s_{53} & s_{54} & s_{55} & s_{56} & s_{57} & s_{58} & v_{5}^{+} \\
s_{61} & s_{62} & s_{63} & s_{64} & s_{65} & s_{66} & s_{67} & s_{68} & v_{6}^{+} \\
s_{71} & s_{72} & s_{73} & s_{74} & s_{75} & s_{76} & s_{77} & s_{78} \\
s_{81} & s_{82} & s_{83} & s_{84} & s_{85} & s_{86} & s_{87} & s_{88} & v_{7}^{+} \\
v_{8}^{+}
\end{array}\right]
$$


Academic Journal of Research and Scientific Publishing | Vol 3 | Issue 31

Publication Date: 5-11-2021 ISSN: 2706-6495

\subsection{Analyzing scattering Matrix}

Utilizing the scattering matrix in analyzing the scattering parameters.

$$
\begin{aligned}
& v_{1}^{-}=s_{11} v_{1}^{+}+s_{12} v_{2}^{+}+s_{13} v_{3}^{+}+s_{14} v_{4}^{+}+s_{15} v_{5}^{+}+s_{16} v_{6}^{+}+s_{17} v_{7}^{+}+s_{18} v_{8}^{+} \\
& v_{2}^{-}=s_{21} v_{1}^{+}+s_{22} v_{2}^{+}+s_{23} v_{3}^{+}+s_{24} v_{4}^{+}+s_{25} v_{5}^{+}+s_{26} v_{6}^{+}+s_{27} v_{7}^{+}+s_{28} v_{8}^{+} \\
& v_{3}^{-}=s_{31} v_{1}^{+}+s_{32} v_{2}^{+}+s_{33} v_{3}^{+}+s_{34} v_{4}^{+}+s_{35} v_{5}^{+}+s_{36} v_{6}^{+}+s_{37} v_{7}^{+}+s_{38} v_{8}^{+} \\
& v_{4}^{-}=s_{41} v_{1}^{+}+s_{42} v_{2}^{+}+s_{43} v_{3}^{+}+s_{44} v_{4}^{+}+s_{45} v_{5}^{+}+s_{46} v_{6}^{+}+s_{47} v_{7}^{+}+s_{48} v_{8}^{+} \\
& v_{5}^{-}=s_{51} v_{1}^{+}+s_{52} v_{2}^{+}+s_{53} v_{3}^{+}+s_{54} v_{4}^{+}+s_{55} v_{5}^{+}+s_{56} v_{6}^{+}+s_{57} v_{7}^{+}+s_{58} v_{8}^{+} \\
& v_{6}^{-}=s_{61} v_{1}^{+}+s_{62} v_{2}^{+}+s_{63} v_{3}^{+}+s_{64} v_{4}^{+}+s_{65} v_{5}^{+}+s_{66} v_{6}^{+}+s_{67} v_{7}^{+}+s_{68} v_{8}^{+} \\
& v_{7}^{-}=s_{71} v_{1}^{+}+s_{72} v_{2}^{+}+s_{73} v_{3}^{+}+s_{74} v_{4}^{+}+s_{75} v_{5}^{+}+s_{76} v_{6}^{+}+s_{77} v_{7}^{+}+s_{78} v_{8}^{+} \\
& v_{8}^{-}=s_{81} v_{1}^{+}+s_{82} v_{2}^{+}+s_{83} v_{3}^{+}+s_{84} v_{4}^{+}+s_{85} v_{5}^{+}+s_{86} v_{6}^{+}+s_{87} v_{7}^{+}+s_{88} v_{8}^{+}
\end{aligned}
$$

\subsection{At center frequency}

$$
\alpha=\frac{-j}{\sqrt{2}} \quad, \quad j \beta=\frac{-1}{\sqrt{2}}
$$

1. $V_{1}^{-}=\alpha^{2} V_{5}^{+}+j \beta \alpha V_{6}^{+}+\alpha j \beta V_{7}^{+}+j \beta^{2} V_{8}^{+}$

$V_{1}^{-}=\frac{-1}{2} V_{5}^{+}+\frac{j}{2} V_{6}^{+}+\frac{j}{2} V_{7}^{+}+\frac{1}{2} V_{8}^{+}$

2. $V_{2}^{-}=\alpha j \beta V_{5}^{+}+j \beta^{2} V_{6}^{+}+\alpha^{2} V_{7}^{+}+j \beta \alpha V_{8}^{+}$

$V_{2}^{-}=\frac{j}{2} V_{5}^{+}+\frac{1}{2} V_{6}^{+}+\frac{-1}{2} V_{7}^{+}+\frac{j}{2} V_{8}^{+}$

3. $V_{3}^{-}=\alpha j \beta V_{5}^{+}+\alpha^{2} V_{6}^{+}+j \beta^{2} V_{7}^{+}+\alpha j \beta V_{8}^{+}$

$V_{3}^{-}=\frac{j}{2} V_{5}^{+}+\frac{-1}{2} V_{6}^{+}+\frac{1}{2} V_{7}^{+}+\frac{j}{2} V_{8}^{+}$

4. $V_{4}^{-}=j \beta^{2} V_{5}^{+}+\alpha j \beta V_{6}^{+}+\alpha j \beta V_{7}^{+}+\alpha^{2} V_{8}^{+}$

$V_{4}^{-}=\frac{1}{2} V_{5}^{+}+\frac{j}{2} V_{6}^{+}+\frac{j}{2} V_{7}^{+}+\frac{-1}{2} V_{8}^{+}$ 
Academic Journal of Research and Scientific Publishing | Vol 3 | Issue 31

Publication Date: 5-11-2021 ISSN: 2706-6495

$$
\begin{gathered}
V_{5}^{-}=\alpha^{2} V_{1}^{+} \alpha j \beta V_{2}^{+}+\alpha j \beta V_{3}^{+}+j \beta^{2} V_{4}^{+} \\
V_{5}^{-}=\frac{-1}{2} V_{1}^{+}+\frac{j}{2} V_{2}^{+}+\frac{j}{2} V_{3}^{+}+\frac{1}{2} V_{4}^{+} \\
V_{6}^{-}=\alpha j \beta V_{1}^{+}+\alpha j \beta V_{2}^{+}+\alpha^{2} V_{3}^{+}+j \beta^{2} V_{4}^{+} \\
V_{6}^{-}=\frac{j}{2} V_{1}^{+}+\frac{j}{2} V_{2}^{+}+\frac{-1}{2} V_{3}^{+}+\frac{1}{2} V_{4}^{+} \\
V_{7}^{-}=\frac{j}{2} V_{1}^{+}+\frac{-1}{2} V_{2}^{+}+\frac{1}{2} V_{3}^{+}+\frac{j}{2} V_{4}^{+} \\
V_{8}^{-}=\frac{1}{2} V_{1}^{+}+\frac{j}{2} V_{2}^{+}+\frac{j}{2} V_{3}^{+}+\frac{-1}{2} V_{4}^{+}
\end{gathered}
$$

\subsection{Scattering matrix at center frequency}

Calculating of the scattering parameter of the proposed 8-port network at the central frequency.

$$
\left[\begin{array}{l}
V_{1}^{-} \\
V_{2}^{-} \\
V_{3}^{-} \\
V_{4}^{-} \\
V_{5}^{-} \\
V_{6}^{-} \\
V_{7}^{-} \\
V_{8}^{-}
\end{array}\right]=\left[\begin{array}{ccccccccc}
0 & 0 & 0 & 0 & \frac{-1}{2} & \frac{j}{2} & \frac{j}{2} & \frac{1}{2} \\
0 & 0 & 0 & 0 & \frac{j}{2} & \frac{1}{2} & \frac{-1}{2} & \frac{j}{2} \\
0 & 0 & 0 & 0 & & & & \\
0 & 0 & 0 & 0 & j & -1 & \frac{1}{2} & \frac{j}{2} \\
\frac{1}{2} & \frac{j}{2} & \frac{j}{2} & \frac{1}{2} & \frac{1}{2} & \frac{1}{2} & \frac{1}{2} & \frac{j}{2} \\
\frac{j}{2} & \frac{j}{2} & \frac{1}{2} & \frac{-1}{2} & \frac{1}{2} & \frac{j}{2} & \frac{j}{2} & \frac{-1}{2} \\
\frac{j}{2} & \frac{-1}{2} & \frac{1}{2} & \frac{j}{2} & 0 & 0 & 0 & 0 & 0 \\
\frac{1}{2} & \frac{j}{2} & \frac{j}{2} & \frac{-1}{2} & 0 & 0 & 0 & 0 \\
V_{3} \\
V_{3}^{+} \\
V_{4}^{+} \\
V_{5}^{+} \\
V_{6}^{+} \\
V_{7}^{+} \\
V_{8}^{+}
\end{array}\right]
$$




\section{Conclusion:}

The novel 8-port network implemented with corrugated (generated slots in both in the coupled area region and the ground plane) have been investigated. The methodology for designing the proposed 8-port network and its constituent couplers is discussed. The properties of the corrugated coupler are shown experimentally to result in an 8-port network implementation with $1 \mathrm{GHz}$ bandwidth. The effect of coupled line separation on the insertion loss in the performance found to be a a $10 \mathrm{~dB}$ improvement by reducing the line separation from 12 mils to 4 mils. The proposed 8-port network have been analyzed, manufactured (see fig.7) and tested with very encouraging result. This proposed network has so many applications in microwaved, millimeter and submillimeter devices (F. Islami, et al, 2017).

\section{Acknowledgement}

This paper would not have been possible without the guidance and the help of several individuals who in one way or another contributed and extended their valuable assistance in the preparation and completion of this study. I would like to express my gratitude to Dr. Khaled Alharbi who undertook to act as my supervisor despite his many other academic and professional commitments. His wisdom, knowledge and commitment to the highest standards inspired and motivated me. Last but not the least, my patents for their continuous support and motivation, the omnipresent God, for answering my prayers for giving me the strength to plod on despite my constitution wanting to give up and throw in the towel, thank you Dear God.

\section{References:}

K. Chang, et al. (1987), on the study of microstrip ring and varactor-tuned ring circuits, IEEE Trans. Microwave Theory and Tech, MTT-35, no. 12, pp. 1288-1295.

G. Matthaei, et al. (1980), Microwave filters, impedance-matching networks, and coupling structures, Artech House.

P. Troughton (1969), Measurement techniques in microstrip, Electronics Letters, vol. 5, no. 2, pp. 25-26. 
C. E. Saavedra (2001) .Microstrip ring resonator using quarter-wave couplers. Electronics Letters, vol. 37, no. 11, pp. 694-695.

J. Sewter and C. E. Saavedra (2002), Microstrip Ring Resonator Using Slow Wave Structure Exhibiting Harmonic Suppression, International Symposium on Antenna Technology and Applied Electromagnetics.

F. Bray, et al. (2013), Global estimates of cancer prevalence for 27 sites in the adult population in 2008, Int. J. Cancer, vol. 132, no. 5, pp. 1133-1145.

M. Miyakawa, et al. (2004), imaging capability of an early stage breast tumor by CP-MCT, Conf. Proc. IEEE Eng. Med. Biol. Soc., vol. 2004, pp. 1427-1430.

E. Praveen, et al. (2016), Design of 1 X8 Phased Array Micro-strip Patch Antennas for Biomedical Applications, no. i. pp. 68-72, 2016.

H. Zhang, et al. (2013), A single antenna based microwave system for breast cancer detection: Experimental results, pp. 477-481.

S. Padhi, et al. (2007), on the accurate modeling of a complex antenna for breast tumor detection using a hybrid MOM/FDTD approach, pp. 6636-6639.

M. T. Islam, et al. (2019), A low cost and portable microwave imaging system for breast tumor detection using UWB directional antenna array, Scientific reports, vol. 9, no. 1, pp. 1-13.

F. Islami, et al. (2017), Global cancer in women: cancer control priorities, Cancer Epidemiology and Prevention Biomarkers, vol. 26, no. 4, pp. 458-470.

Copyright @ 2021 Eng. W. M. ALSAMKARI, Prof. ADNAN. M. AFFANDI, AJRSP. This is an Open-Access Article Distributed under the Terms of the Creative Commons Attribution License (CC BY NC)

Doi: doi.org/10.52132/Ajrsp.e.2021.31.1 